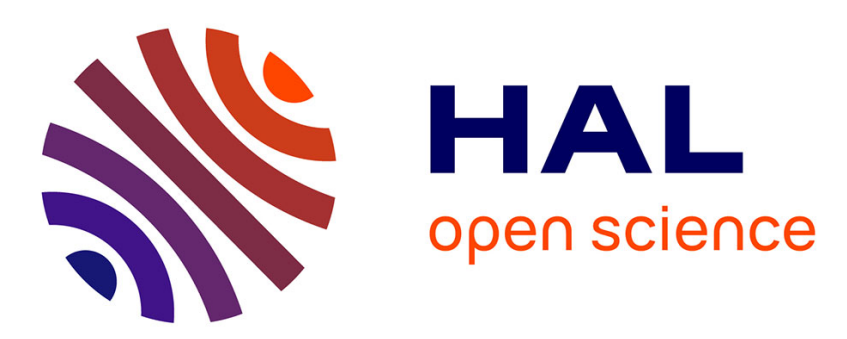

\title{
Average State Estimation in Large-scale Clustered Network Systems
}

Muhammad Umar B. Niazi, Carlos Canudas de Wit, Alain Kibangou

\section{To cite this version:}

Muhammad Umar B. Niazi, Carlos Canudas de Wit, Alain Kibangou. Average State Estimation in Large-scale Clustered Network Systems. IEEE Transactions on Control of Network Systems, 2020, 7 (4), pp.1736 - 1745. 10.1109/TCNS.2020.2999304 . hal-02524982

\section{HAL Id: hal-02524982 \\ https://hal.science/hal-02524982}

Submitted on 30 Mar 2020

HAL is a multi-disciplinary open access archive for the deposit and dissemination of scientific research documents, whether they are published or not. The documents may come from teaching and research institutions in France or abroad, or from public or private research centers.
L'archive ouverte pluridisciplinaire $\mathbf{H A L}$, est destinée au dépôt et à la diffusion de documents scientifiques de niveau recherche, publiés ou non, émanant des établissements d'enseignement et de recherche français ou étrangers, des laboratoires publics ou privés. 


\title{
Average State Estimation in Large-scale Clustered Network Systems
}

\author{
Muhammad Umar B. Niazi, Carlos Canudas-de-Wit, and Alain Y. Kibangou
}

\begin{abstract}
For the monitoring of large-scale clustered network systems (CNS), it suffices in many applications to know the aggregated states of given clusters of nodes. This paper provides necessary and sufficient conditions such that the average states of the pre-specified clusters can be reconstructed and/or asymptotically estimated. To achieve computational tractability, the notions of average observability (AO) and average detectability (AD) of the CNS are defined via the projected network system, which is of tractable dimension and is obtained by aggregating the clusters. The corresponding necessary and sufficient conditions of AO and $A D$ are provided and interpreted through the underlying structure of the induced subgraphs and the induced bipartite subgraphs, which capture the intra-cluster and inter-cluster topologies of the CNS, respectively. Moreover, the design of an average state observer whose dimension is minimum and equals the number of clusters in the CNS is presented.
\end{abstract}

Index Terms-Clustered network systems, average observability, average detectability, average state observer.

\section{INTRODUCTION}

State reconstruction of large-scale network systems is challenging because of limited computational and sensing resources, [1]. However, knowing the entire state vector is often unnecessary for monitoring purposes, and it suffices to reconstruct some aggregated states of a system, [2]-[4]. In this paper, we study the reconstruction and estimation of the average states of given clusters (or sectors) of nodes in a network system, which is meaningful in many applications such as urban traffic networks [5], building thermal systems [6], epidemic spread over networks [7], and power grids [8].

We consider a large-scale clustered network system (CNS) with few measured nodes-the nodes where sensors are placed. The remaining unmeasured nodes are partitioned into multiple clusters. The dimension of a CNS can be very large, which presents computational difficulties. Therefore, by aggregating the clusters of nodes, we obtain a projected network system (PNS), which is of tractable dimension and provides the dynamics of the average states of the clusters. In this regard, [9] is among the earliest to study the aggregated dynamics of large-scale systems. Later, [10] builds on [9] and introduces the notion of exact lumpability, which has several interpretations. Algebraically, a linear time-invariant network

M. U. B. Niazi and C. Canudas-de-Wit are with CNRS, GIPSA-Lab, Grenoble, France. (email: muhammad-umar-b.niazi@gipsa-lab.fr, carlos.canudas-dewit@gipsa-lab.fr)

A. Y. Kibangou is with Univ. Grenoble Alpes, CNRS, Inria, Grenoble INP, GIPSA-Lab, Grenoble, France. (email: alain.kibangou@ univ-grenoblealpes.fr)

This work is supported by European Research Council (ERC) under the European Union's Horizon 2020 research and innovation programme (ERCAdG no. 694209, Scale-FreeBack, website: http://scale-freeback.eu/). system is exactly lumpable if and only if the projection is $A$ invariant, [11], where $A$ is the state matrix of the network system. Graph theoretically, this condition of $A$-invariance is satisfied only if the clusters of a network system are chosen according to an (almost) equitable partition, [12]-[16]. However, achieving exact lumpability by network partitioning is quite difficult, as remarked in [17]. This is due to the constraints on sensor locations and number of clusters, and, in physical network systems, the clusters are also required to be connected, [18]. Therefore, in general, the PNS is influenced by a vector of average deviation of the clusters, which can be considered as a structured unknown input.

Average state estimation by clustering-based model reduction technique [19] has been studied in [20]. The advantage of this approach is that the states of the reduced system approximate the average states of the network clusters. Thus, it suffices to design an average observer by employing the reduced system. However, this approach becomes irrelevant when the clusters are a priori specified. In such a case, we aim to provide conditions under which the average states of the clusters can be reconstructed and/or asymptotically estimated.

First, we study average observability, a notion that ensures the reconstruction of the average states of clusters from the dedicated state measurements at the measured nodes. Average observability falls under the notion of functional observability, [21]-[23], which concerns the reconstruction of a set of linear functionals of the state. However, the approach followed in [21]-[23] is computationally intractable for large-scale systems. Therefore, we define average observability via the PNS, which allows for computationally tractable necessary and sufficient conditions. We also provide a graph-theoretic interpretation for average observability through the induced bipartite subgraph that captures the topology between the measured and the clusters of unmeasured nodes. By assuming average observability, we provide a necessary and sufficient condition for an average state observer that asymptotically estimates the average states of clusters at an arbitrary rate.

Second, we study average detectability, a notion that ensures the asymptotic stability of the average states of clusters. We show that average detectability allows for the open-loop estimation of the average states. We provide a graph-theoretic interpretation of average detectability through the structure of induced subgraphs and induced bipartite subgraphs formed by the clusters of unmeasured nodes. These subgraphs capture the intra-cluster and the inter-cluster graph topology of the network system, respectively.

In summary, average observability mainly concerns the graph topology between the unmeasured and measured nodes, 
and average detectability relates to the graph topology of the clusters of unmeasured nodes. The initial investigation of average observability and average detectability for a single cluster of unmeasured nodes is presented in [24]. Here, we extend the results to multiple clusters and also devise average observers for the asymptotic estimation of the average states.

\section{PRoblem Formulation}

We provide the preliminaries leading to the problem statement in this section. First, we define a clustered network system (CNS), then, by aggregating the clusters, we define the projected network system (PNS).

\section{A. Notations}

The sets of real and complex numbers are denoted as $\mathbb{R}$ and $\mathbb{C}$, respectively. Vectors are denoted by bold lowercase letters and matrices by uppercase letters. A column vector of ones and an identity matrix are denoted as $\mathbf{1}_{k}$ and $I_{k}$, respectively, where the subscript $k$ (sometimes omitted for brevity) indicates their dimensions. The standard basis vector $\mathbf{e}_{v} \in \mathbb{R}^{k}$ is the $v$-th column of $I_{k}$. We denote by $[A]_{i j}$ the $i j$-entry of a matrix $A \in \mathbb{R}^{n \times m}$. When $A \in \mathbb{R}^{n \times n}$ is a square matrix, $\operatorname{eig}(A) \subset \mathbb{C}$ denotes the set of eigenvalues of $A$. By $\operatorname{diag}\left[A_{1}, \cdots, A_{k}\right]$ we denote a block diagonal matrix with $A_{1}, \cdots, A_{k}$ at its diagonal blocks. The Euclidean norm is denoted by $\|\cdot\|$.

\section{B. Clustered network system}

Consider a network system represented by a weighted digraph $\mathcal{G}=(\mathcal{V}, \mathcal{E})$ with the set of nodes $\mathcal{V}=\{1, \cdots, n\}$ and the ordered set of edges $\mathcal{E} \subseteq \mathcal{V} \times \mathcal{V}$. The edge $(i, j)$ is a directed arc from node $j$ to $i$, i.e., $i \leftarrow j$, with a weight $a_{i j}>0$ associated to it. The state of each node evolves according to

$$
\dot{x}_{i}(t)=a_{i i} x_{i}(t)+\sum_{j \in \mathcal{N}_{i}^{\downarrow}} a_{i j} x_{j}(t)+\sum_{l=1}^{p} b_{i l} u_{l}(t),
$$

where $x_{i}(t) \in \mathbb{R}$, for $i \in \mathcal{V}$, are the states; $u_{l}(t) \in \mathbb{R}$, for $l=1, \cdots, p$, are the known external inputs; and $\mathcal{N}_{i}^{\downarrow}:=\{j \in \mathcal{V}:(i, j) \in \mathcal{E}, j \neq i\}$ is the set of $i$ 's inneighbors. The first term on the right-hand side of (1) refers to the local feedback at each node $i$ with the weight $a_{i i} \leq 0$. The second term corresponds to the aggregated inflow from $j \in \mathcal{N}_{i}^{\downarrow}$ to $i$ weighted by $a_{i j}>0$, respectively. The third term depicts the inputs at $i$ weighted by $b_{i l} \in \mathbb{R}$, respectively.

Remark 1: The local feedback corresponds to the selfloops in the graph, which are omitted from the figures in this paper and are considered implicit. We further remark that the self-loop weights $a_{i i}$, for all $i \in \mathcal{V}$, considered in (1) are quite general. There might be a network structure associated to these scalars as in consensus-seeking multiagent systems [16], reaction-diffusion systems [19], and linear compartmental systems [25].

By considering $\mathbf{x}(t)=\left[x_{1}(t) \cdots x_{n}(t)\right]^{\mathrm{T}} \in \mathbb{R}^{n}$ as a state vector and $\mathbf{u}(t)=\left[u_{1}(t) \cdots u_{p}(t)\right]^{\mathrm{T}} \in \mathbb{R}^{p}$ as an input vector, we have

$$
\boldsymbol{\Sigma}:\left\{\begin{aligned}
\dot{\mathbf{x}}(t) & =A \mathbf{x}(t)+B \mathbf{u}(t) \\
\mathbf{y}(t) & =C \mathbf{x}(t)
\end{aligned}\right.
$$

where $\mathbf{y}(t) \in \mathbb{R}^{k}$ is the output vector. The system $\boldsymbol{\Sigma}$ is a large-scale system, i.e., the number of nodes $n$ is very large, where the state matrix $A \in \mathbb{R}^{n \times n}$ is given by

$$
[A]_{i j}=\left\{\begin{array}{cl}
a_{i j}, & \text { if }(i, j) \in \mathcal{E} \text { and } i \neq j \\
a_{i i}, & \text { if } i=j \\
0, & \text { otherwise }
\end{array}\right.
$$

The input matrix $B \in \mathbb{R}^{n \times p}$ contains the weights of the input configurations, namely $[B]_{i l}=b_{i l} \in \mathbb{R}$ for all $i \in \mathcal{V}$ and $l=1, \cdots, p$. The output vector $\mathbf{y}(t) \in \mathbb{R}^{k}$ contains the measurements of the dedicated sensors at $k$ nodes, which are called the measured nodes. Thus the output matrix $C \in \mathbb{R}^{k \times n}$

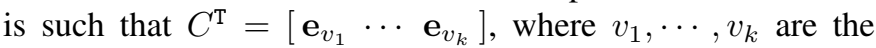
measured nodes and $\mathbf{e}_{v} \in \mathbb{R}^{n}$ is the $v$-th standard basis vector. The remaining nodes are called the unmeasured nodes. We assume $k<m$, where $m=n-k$ is the number of unmeasured nodes. Also, the pair $(C, A)$ need not be observable.

Suppose the nodes $\mathcal{V}$ are partitioned into $r$ disjoint clusters, where $2 \leq r<m$. That is, we are given a clustering (or partition) $\mathcal{P}=\left\{\mathcal{C}_{1}, \cdots, \mathcal{C}_{r}\right\}$ of the network nodes such that $\mathcal{V}=\mathcal{C}_{1} \cup \cdots \cup \mathcal{C}_{r}$ and, for any $p, q=1, \cdots, r$ and $p \neq q$, $\mathcal{C}_{p} \cap \mathcal{C}_{q}=\emptyset$. Let $\mathcal{G}_{p p}=\left(\mathcal{C}_{p}, \mathcal{E}_{p p}\right)$ be an induced subgraph, where $\mathcal{E}_{p p}=\mathcal{E} \cap\left(\mathcal{C}_{p} \times \mathcal{C}_{p}\right)$ is the ordered set of edges within $\mathcal{G}_{p p}$. The subgraph $\mathcal{G}_{p p}$ captures the intra-cluster graph structure of the cluster $\mathcal{C}_{p}$. The inter-cluster graph structure, on the other hand, is captured by an induced bipartite subgraph $\mathcal{G}_{p q}=\left(\mathcal{C}_{p}, \mathcal{C}_{q}, \mathcal{E}_{p q}\right)$, where $\mathcal{E}_{p q}=\mathcal{E} \cap\left(\mathcal{C}_{p} \times \mathcal{C}_{q}\right)$ is the ordered set of directed edges from $\mathcal{C}_{q}$ to $\mathcal{C}_{p}$ with $p \neq q$. Here, and in what follows, the subscripts $p, q=1, \cdots, r$.

Without loss of generality, we suppose that $\mathcal{C}_{1}$ is the set of measured nodes and the nodes $\mathcal{V}$ are ordered such that $\mathcal{C}_{1}=\{1, \cdots, k\}, \mathcal{C}_{2}=\left\{k+1, \cdots, k+m_{2}\right\}$, and so on; where $\left|\mathcal{C}_{h}\right|=m_{h}$, for $h=2, \cdots, r$, and $\sum_{h=2}^{r} m_{h}=n-k$. Then, the state vector of the network system $\boldsymbol{\Sigma}$ is partitioned as $\mathbf{x}(t)=\left[\mathbf{x}_{1}^{\mathrm{T}}(t) \cdots \mathbf{x}_{r}^{\mathrm{T}}(t)\right]^{\mathrm{T}} \in \mathbb{R}^{n}$, where $\mathbf{x}_{1}(t) \in \mathbb{R}^{k}$ is the state of measured nodes and $\mathbf{x}_{h}(t) \in \mathbb{R}^{m_{h}}$ is the state of the unmeasured nodes in $\mathcal{C}_{h}$ for $h=2, \cdots, r$. In what follows, the subscripts $h, g=2, \cdots, r$.

The clustered network system (CNS) is defined as $\boldsymbol{\Sigma}$ with clustering $\mathcal{P}$, where the system matrices are partitioned as

$$
\begin{aligned}
A & =\left[\begin{array}{cc}
A_{11} & \tilde{A}_{12} \\
\tilde{A}_{21} & \tilde{A}_{22}
\end{array}\right], \quad B=\left[\begin{array}{c}
B_{1} \\
\tilde{B}_{2}
\end{array}\right], \\
C & =\left[\begin{array}{cc}
I_{k} & 0
\end{array}\right]
\end{aligned}
$$

with $\tilde{B}_{2}=\left[\begin{array}{lll}B_{2}^{\mathrm{T}} & \cdots & B_{r}^{\mathrm{T}}\end{array}\right]^{\mathrm{T}}, \tilde{A}_{12}=\left[\begin{array}{lll}A_{12} & \cdots & A_{1 r}\end{array}\right]$,

$$
\tilde{A}_{21}=\left[\begin{array}{c}
A_{21} \\
\vdots \\
A_{r 1}
\end{array}\right], \quad \tilde{A}_{22}=\left[\begin{array}{ccc}
A_{22} & \cdots & A_{2 r} \\
\vdots & \ddots & \vdots \\
A_{r 2} & \cdots & A_{r r}
\end{array}\right]
$$

The submatrices $A_{p p}$ and $A_{p q}$ contain the weighted edge configurations of the induced subgraphs $\mathcal{G}_{p p}$ and the induced bipartite subgraphs $\mathcal{G}_{p q}$, respectively, for $p \neq q$. 


\section{Projected network system}

Let $\mathcal{Q}=\left\{\mathcal{C}_{2}, \cdots, \mathcal{C}_{r}\right\} \subset \mathcal{P}$ be the partition of unmeasured nodes. The characteristic matrix of $\mathcal{Q}$, [26], is defined as $Q=\operatorname{diag}\left[\mathbf{1}_{m_{2}}, \cdots, \mathbf{1}_{m_{r}}\right]$. Let the projection matrices be

$$
P=\left[\begin{array}{cc}
I_{k} & 0 \\
0 & \Lambda Q^{\mathrm{T}}
\end{array}\right] \text { and } P^{+}=\left[\begin{array}{cc}
I_{k} & 0 \\
0 & Q
\end{array}\right]
$$

where $\Lambda=\left(Q^{\mathrm{T}} Q\right)^{-1}=\operatorname{diag}\left[\frac{1}{m_{2}}, \cdots, \frac{1}{m_{r}}\right]$ and $P P^{+}=I$. Let $\mathbf{z}(t)=P \mathbf{x}(t)=\left[\begin{array}{llll}\mathbf{x}_{1}^{\mathrm{T}}(t) & x_{2}^{\mathrm{av}}(t) & \cdots & x_{r}^{\mathrm{av}}(t)\end{array}\right]^{\mathrm{T}} \in \mathbb{R}^{\ell}$ be the projected state, where

$$
x_{h}^{\mathrm{av}}(t)=\frac{1}{m_{h}} \mathbf{1}^{\mathrm{T}} \mathbf{x}_{h}(t)
$$

is the average state of the cluster $\mathcal{C}_{h}$ and $\ell=k+r-1$. Note that $\mathbf{x}(t)=P^{+} \mathbf{z}(t)+\left(I-P^{+} P\right) \mathbf{x}(t)$, [27], where

$$
\left(I-P^{+} P\right) \mathbf{x}(t)=\left[\begin{array}{l}
0 \\
J
\end{array}\right] \mathbf{x}(t)
$$

and $J=\left[\begin{array}{ll}0 & I_{m}-Q \Lambda Q^{\mathrm{T}}\end{array}\right]$. The vector $\boldsymbol{\sigma}(t):=J \mathbf{x}(t) \in \mathbb{R}^{m}$ is called the average deviation vector, which is given as $\boldsymbol{\sigma}(t)=\left[\boldsymbol{\sigma}_{2}^{\mathrm{T}}(t) \cdots \boldsymbol{\sigma}_{r}^{\mathrm{T}}(t)\right]^{\mathrm{T}}$, where $\boldsymbol{\sigma}_{h}(t):=\mathbf{x}_{h}(t)-\mathbf{1} x_{h}^{\mathrm{av}}(t)$.

We project the state dynamics of $\boldsymbol{\Sigma}$ on a lower-dimensional state space $\mathbb{R}^{\ell}$, where $\ell<n$, and obtain the projected network system (PNS)

$$
\dot{\Sigma}: \begin{cases}\dot{\mathbf{z}}(t) & =E \mathbf{z}(t)+F \boldsymbol{\sigma}(t)+G \mathbf{u}(t) \\ 0 & =Q^{\mathrm{T}} \boldsymbol{\sigma}(t), \\ \mathbf{y}(t) & =H \mathbf{z}(t),\end{cases}
$$

where $E=P A P^{+}, F \boldsymbol{\sigma}(t)=P A\left(I-P^{+} P\right) \mathbf{x}(t), G=P B$, and $H=C P^{+}$with

$$
\begin{aligned}
& E=\left[\begin{array}{cc}
A_{11} & \tilde{A}_{12} Q \\
\Lambda Q^{\mathrm{T}} \tilde{A}_{21} & \Lambda Q^{\mathrm{T}} \tilde{A}_{22} Q
\end{array}\right], \quad F=\left[\begin{array}{c}
\tilde{A}_{12} \\
\Lambda Q^{\mathrm{T}} \tilde{A}_{22}
\end{array}\right], \\
& H=\left[\begin{array}{cc}
I_{k} & 0
\end{array}\right], \quad G=\left[\begin{array}{c}
B_{1} \\
\Lambda Q^{\mathrm{T}} \tilde{B}_{2}
\end{array}\right] .
\end{aligned}
$$

Remark 2: Since the average deviation vector $\boldsymbol{\sigma}(t)$ is the function of unmeasured part of the state $\mathbf{x}(t)$, we consider $\boldsymbol{\sigma}(t)$ to be an unknown input in $\boldsymbol{\Sigma}^{\circ}$ with the structural property $Q^{\mathrm{T}} \boldsymbol{\sigma} \equiv 0$. This doesn't change the definition that $\boldsymbol{\sigma}=J \mathbf{x}$. Moreover, note that the output $\mathbf{y}(t)$ in $\mathbf{\Sigma}^{\circ}$ is same as in $\boldsymbol{\Sigma}$. This is because the projection $P$ only aggregates the clusters of unmeasured nodes $\mathcal{C}_{h}$, and not the cluster of measured nodes $\mathcal{C}_{1}$, thus we have that $C \mathbf{x}(t)=H \mathbf{z}(t)$ since $C\left(I-P^{+} P\right)=0$. This aggregation process is illustrated in Fig. 1, where the black nodes are measured and the given clusters of unmeasured nodes are aggregated and treated as a single (super) node. $\diamond$

The purpose of $\dot{\Sigma}^{\circ}$ is to attain computational feasibility since the dimension of its state space is much lower than that of $\boldsymbol{\Sigma}$. In our analysis, we don't consider the dynamics of $\boldsymbol{\sigma}(t)$ and only consider its structural property. That is, $\boldsymbol{\sigma}=J_{\mathbf{x}}$ is unknown, however, it satisfies $Q^{\mathrm{T}} \boldsymbol{\sigma} \equiv 0$, which is the only known information about $\boldsymbol{\sigma}$. Even though considering the dynamics $\dot{\boldsymbol{\sigma}}(t)$ may allow for more information about the system, however, it will make $\stackrel{\circ}{\Sigma}$ a transformed system whose state evolves in higher dimension than $\boldsymbol{\Sigma}$. In such a case, the computational tractability cannot be achieved for large-scale systems.
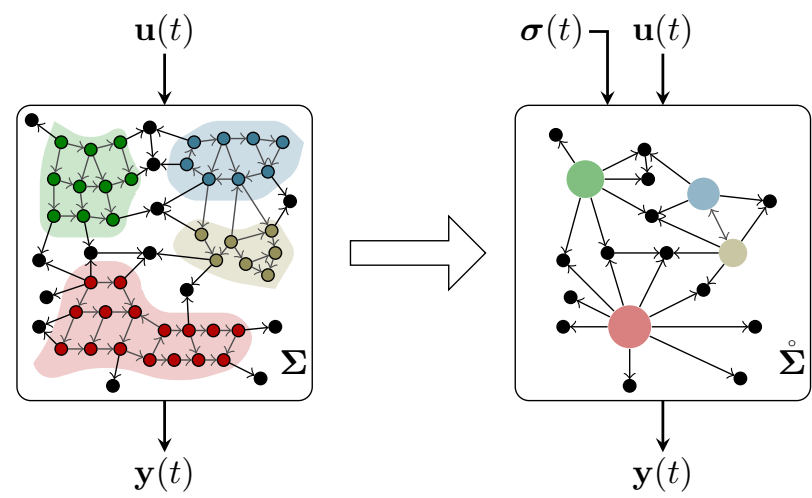

Fig. 1: Obtaining the PNS $\dot{\Sigma}^{\Sigma}$ from the CNS $\boldsymbol{\Sigma}$ by aggregating the clusters of unmeasured nodes. Here, $\boldsymbol{\sigma}(t)$ is the average deviation vector which is considered as an unknown input satisfying $Q^{\mathrm{T}} \boldsymbol{\sigma} \equiv 0$. Note that both systems yield the same output $\mathbf{y}(t)$, i.e., the sensor measurements at black nodes.

We define outflow centrality of $i$ as $\phi_{i}=a_{i i}+\sum_{j \in \mathcal{N}_{i}^{\uparrow}} a_{j i}$, where $\mathcal{N}_{i}^{\uparrow}:=\{j \in \mathcal{V}:(j, i) \in \mathcal{E}, j \neq i\}$ is the set of node $i$ 's out-neighbors. Then, the internal outflow centrality of $i \in \mathcal{C}_{h}$ in the induced subgraph $\mathcal{G}_{h h}$ is computed as

$$
\phi_{i, h}=a_{i i}+\sum_{j \in \mathcal{N}_{i, h}^{\uparrow}} a_{j i},
$$

where $\mathcal{N}_{i, h}^{\uparrow}=\mathcal{N}_{i}^{\uparrow} \cap \mathcal{C}_{h}$ are the internal out-neighbors of $i \in \mathcal{C}_{h}$. The relative out-degree of $i \in \mathcal{C}_{q}$ in the induced bipartite subgraph $\mathcal{G}_{p q}$ is computed as

$$
d_{i, p}=\sum_{j \in \mathcal{N}_{i, p}^{\uparrow}} a_{j i}
$$

where $p \neq q$ and $\mathcal{N}_{i, p}^{\uparrow}=\mathcal{N}_{i}^{\uparrow} \cap \mathcal{C}_{p}$.

We further remark that $\Sigma_{\Sigma}^{\circ}$ only requires the aggregated information about the network. That is, apart from the induced subgraphs $\mathcal{G}_{11}$ and $\mathcal{G}_{1 h}$, i.e., the matrices $A_{11}$ and $A_{1 h}$, we require the internal outflow centrality of nodes in $\mathcal{C}_{h}$ in the induced subgraphs $\mathcal{G}_{h h}$, i.e., the entries of $\mathbf{1}_{m_{h}}^{\mathrm{T}} A_{h h}$, and the relative out-degree of nodes in $\mathcal{C}_{q}$ in the induced bipartite subgraphs $\mathcal{G}_{p q}$ for $p \neq q$, i.e., the entries of $\mathbf{1}_{m_{p}}^{\mathrm{T}} A_{p q}$.

\section{Problem statement}

Given a CNS $\boldsymbol{\Sigma}$ with the set of measured nodes $\mathcal{C}_{1}$ and the clusters $\mathcal{C}_{2}, \cdots, \mathcal{C}_{r}$ of unmeasured nodes, we investigate the following:

(P1) Is it possible to reconstruct the average states $x_{h}^{\mathrm{av}}(t)$ from PNS $\Sigma$, for $h=2, \cdots, r$, by using the measurements of the dedicated sensors $\mathbf{y}(t)$ and the knowledge of the external input $\mathbf{u}(t)$ ?

(P2) Do the average states $x_{h}^{\mathrm{av}}(t)$ converge to 0 as $t \rightarrow \infty$ if $\mathbf{y}(t)=0$ and $\mathbf{u}(t)=0$ for all $t \geq 0$ ?

The problem (P1) concerns the observability of average states of given clusters, which is called average observability of the CNS, and (P2) concerns the detectability of average states of given clusters, which is called average detectability of the CNS. 


\section{AVERAGE OBSERVABILITy}

The resulting problem of interest in a CNS is the reconstruction of the average states of given clusters $\mathcal{C}_{2}, \cdots, \mathcal{C}_{r}$. We address this reconstruction problem through the PNS to attain computational feasibility. In other words, we define the average observability of $\boldsymbol{\Sigma}$ via the observability of $\dot{\Sigma}$.

The CNS $\boldsymbol{\Sigma}$ with a given set $\mathcal{C}_{1}$ of $k$ measured nodes and a clustering $\mathcal{Q}=\left\{\mathcal{C}_{2}, \cdots, \mathcal{C}_{r}\right\}$ of $m$ unmeasured nodes is said to be average observable ( $\mathrm{AO})$ if it is possible to reconstruct the average states $x_{2}^{\mathrm{av}}(t), \cdots, x_{r}^{\mathrm{av}}(t)$ of the clusters from the PNS $\Sigma^{\circ}$ using the knowledge of the output $\mathbf{y}(t)$ and the input $\mathbf{u}(t)$, for all $t \geq 0$. For reconstructing the average states from $\stackrel{\circ}{\Sigma}$, it is necessary and sufficient that $\dot{\Sigma}$ is observable. Thus, the

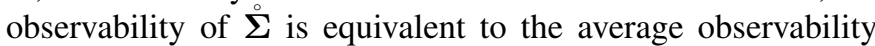
of $\boldsymbol{\Sigma}$ by definition.

To put it precisely, suppose $\mathbf{u} \equiv 0$ (since it is a known input and can be subtracted from the output) and consider the output trajectory from $\Sigma^{\circ}$

$\mathbf{y}_{\boldsymbol{\sigma}}\left(t, \mathbf{z}_{0}\right)=H\left(\exp (E t) \mathbf{z}_{0}+\int_{0}^{t} \exp [E(t-\tau)] F \boldsymbol{\sigma}(\tau) d \tau\right)$,

where $\mathbf{z}_{0}=\mathbf{z}(0)$ is the initial state of $\stackrel{\circ}{\boldsymbol{\Sigma}}$. Then, $\boldsymbol{\Sigma}$ is AO if, for all $t \geq 0$ and $\mathbf{z}_{0} \in \mathbb{R}^{\ell}, \mathbf{y}_{\boldsymbol{\sigma}}\left(t, \mathbf{z}_{0}\right)=0$ implies that $x_{h}^{\text {av }}(t)=0$, for all $h=2, \cdots, r$, where $\ell=k+r-1$ and $Q^{\mathrm{T}} \boldsymbol{\sigma} \equiv 0$.

The definition given above matches the definition of strong observability in the presence of arbitrary unknown inputs, see [28] and [29, section 7.5]. In our case, however, the unknown input $\boldsymbol{\sigma}(t)$ in $\stackrel{\circ}{\boldsymbol{\Sigma}}$ satisfies the structural property $Q^{\mathrm{T}} \boldsymbol{\sigma} \equiv 0$, which means that $\boldsymbol{\sigma}: \mathbb{R}_{>0} \rightarrow \operatorname{ker}\left(Q^{\mathrm{T}}\right)$. Notice that a set of such signals includes the originally defined $\boldsymbol{\sigma}(t)=J \mathbf{x}(t)$, because the columns of $J$ form a complete basis of $\operatorname{ker}\left(Q^{\mathrm{T}}\right)$. This implies that the property $Q^{\mathrm{T}} \boldsymbol{\sigma} \equiv 0$ is independent of $\mathbf{x}(t) \in \mathbb{R}^{n}$ for all $t \geq 0$, which is the essential fact used in proving the necessity of results in this paper.

\section{A. Necessary and sufficient conditions}

In this subsection, we first state a necessary and sufficient condition for AO of $\boldsymbol{\Sigma}$. Then, we derive a necessary condition and a sufficient condition based on the structure of $\boldsymbol{\Sigma}$.

Proposition 1: The CNS $\boldsymbol{\Sigma}$ is average observable if and only if there exists a matrix $N \in \mathbb{R}^{\ell \times \ell}$ such that the following two conditions hold:

(i) $N F \boldsymbol{\sigma}(t)=0$ for all $t \geq 0$.

(ii) $\operatorname{rank}\left[\begin{array}{c}s N-N E \\ H\end{array}\right]=\ell$ for all $s \in \mathbb{C}$.

Here, $E \in \mathbb{R}^{\ell \times \ell}, F \in \mathbb{R}^{\ell \times m}$ and $H \in \mathbb{R}^{k \times \ell}$ are given in (3), and $\boldsymbol{\sigma}(t) \in \mathbb{R}^{m}$ satisfies $Q^{\mathrm{T}} \boldsymbol{\sigma} \equiv 0$.

Proof: (Sufficiency) By multiplying the state equation of $\dot{\Sigma}^{\circ}$ by $N \in \mathbb{R}^{\ell \times \ell}$, we obtain a descriptor system

$$
\stackrel{\circ}{\mathbf{\Sigma}}_{N}:\left\{\begin{array}{l}
N \dot{\mathbf{z}}(t)=N E \mathbf{z}(t)+N F \boldsymbol{\sigma}(t)+N G \mathbf{u}(t) \\
\mathbf{y}(t)=H \mathbf{z}(t) .
\end{array}\right.
$$

Assume $N$ is such that (i) holds, then, according to [30] and [31], $\dot{\Sigma}_{N}$ is observable if and only if (ii) holds. Therefore, if both (i) and (ii) hold, we can reconstruct $\mathbf{z}(t)=\left[\begin{array}{llll}\mathbf{x}_{1}^{\mathrm{T}}(t) & x_{2}^{\mathrm{av}}(t) & \cdots & x_{r}^{\mathrm{av}}(t)\end{array}\right]^{\mathrm{T}}$ from $\mathbf{\Sigma}_{N}$.
(Necessity) To prove the necessity of (i) and (ii), we need to show that if (i) and/or (ii) do not hold, then $\boldsymbol{\Sigma}$ is not AO. If we show that $\boldsymbol{\Sigma}$ is not AO if (i) 'or' (ii) does not hold, then it follows that $\Sigma$ is not AO if (i) 'and' (ii) do not hold. Therefore, in the following, we address only the two cases:

Case 1 - (i) doesn't hold: Since (i) doesn't hold, therefore, to reconstruct the average states from $\dot{\Sigma}^{\circ}$, it is necessary and sufficient, according to [28], to satisfy

$$
\operatorname{rank}\left[\begin{array}{cc}
s_{0} I-E & F \\
H & 0
\end{array}\right]=\ell+\operatorname{rank} F, \forall s_{0} \in \mathbb{C} .
$$

But (6) does not hold for $s_{0}=0$, i.e.,

$$
\begin{aligned}
\operatorname{rank}\left[\begin{array}{cc}
s_{0} I-E & F \\
H & 0
\end{array}\right] & =\operatorname{rank}\left[\begin{array}{ccc}
-E_{1} & -F Q & F \\
I_{k} & 0 & 0
\end{array}\right] \\
& <\ell+\operatorname{rank} F
\end{aligned}
$$

where $E_{1}=\left[\begin{array}{c}A_{11} \\ \Lambda Q^{\mathrm{T}} \tilde{A}_{21}\end{array}\right]$ and $E=\left[\begin{array}{ll}E_{1} & F Q\end{array}\right]$ from (3). This is because $\operatorname{rank}\left[\begin{array}{ll}-F Q & F\end{array}\right]<\operatorname{rank}(F Q)+\operatorname{rank}(F)$. Therefore, the average states cannot be reconstructed from $\dot{\Sigma}^{\circ}$.

Case 2 - (ii) doesn't hold: If there exists $N$ such that (i) holds, then we have $\dot{\Sigma}_{N}$. Since (ii) doesn't hold, therefore, according to [30]-[32], $\mathbf{\Sigma}_{N}$ is not observable. Therefore, $x_{2}^{\text {av }}(t), \cdots, x_{r}^{\text {av }}(t)$ cannot be reconstructed.

We have shown that $\boldsymbol{\Sigma}$ is not AO if (i) and/or (ii) doesn't hold. Adding this with the sufficiency part, it is proved that $\boldsymbol{\Sigma}$ is AO if and only if both (i) and (ii) hold.

Based on Proposition 1(i), we provide the following necessary condition of average observability.

Theorem 2 (Necessary condition): The CNS $\boldsymbol{\Sigma}$ is average observable only if

$$
\operatorname{rank}\left[\begin{array}{c}
F \\
Q^{\mathrm{T}}
\end{array}\right]=\operatorname{rank} F
$$

where $Q \in \mathbb{R}^{m \times(r-1)}$ is the characteristic matrix of the clustering $\mathcal{Q}$ of unmeasured nodes and $F$ is given in (3).

Proof: First, note that $\boldsymbol{\sigma}: \mathbb{R}_{\geq 0} \rightarrow \operatorname{ker}\left(Q^{\mathrm{T}}\right)$ since $\boldsymbol{\sigma}=J \mathbf{x}$, where $J=\left[\begin{array}{ll}0 & I-Q \Lambda Q^{\mathrm{T}}\end{array}\right]$. The columns of $I-Q \Lambda Q^{\mathrm{T}}$ form the basis of $\operatorname{ker}\left(Q^{\mathrm{T}}\right)$ because $Q^{\mathrm{T}}\left(I-Q \Lambda Q^{\mathrm{T}}\right)=0$ and $\operatorname{rank}\left(I-Q \Lambda Q^{\mathrm{T}}\right)=m-(r-1)$, which equals the dimension of $\operatorname{ker}\left(Q^{\mathrm{T}}\right)$ since $\operatorname{rank}\left(Q^{\mathrm{T}}\right)=r-1$ and $Q^{\mathrm{T}} \in \mathbb{R}^{(r-1) \times m}$ with $m \geq r-1$. Second, note that (8) is equivalent to the existence of $N \in \mathbb{R}^{\ell \times \ell}$ such that $N F=V Q^{\mathrm{T}}$, where $V \in \mathbb{R}^{\ell \times(r-1)}$ is a nonzero arbitrary matrix. That is, we can obtain the $\ell$ rows of $V Q^{\mathrm{T}}$ by performing $\ell$ elementary row operations on $F$ if and only if (8) holds. Finally, to prove the necessity of (8), we prove that it is equivalent to Proposition 1(i):

Suppose (8) holds, then there exists $N \in \mathbb{R}^{\ell \times \ell}$ such that $N F=V Q^{\mathrm{T}}$. Thus, $N F \boldsymbol{\sigma}(t)=V Q^{\mathrm{T}} \boldsymbol{\sigma}(t)=0$ for all $t \geq 0$. In the other direction, suppose there exists $N \in \mathbb{R}^{\ell \times \ell}$ such that $N F \boldsymbol{\sigma}(t)=0$ for all $t \geq 0$. Then, it holds that $\operatorname{ker}(N F) \supseteq \operatorname{ker}\left(Q^{\mathrm{T}}\right)$. Hence, there exists $V \in \mathbb{R}^{\ell \times(r-1)}$ such that $N F=V Q^{\mathrm{T}}$.

Notice that (8) is not sufficient for AO of $\boldsymbol{\Sigma}$ because it may not satisfy Proposition 1(ii). We provide the following 
sufficient condition, which has an intuitive graph-theoretic interpretation.

Theorem 3 (Sufficient condition): The CNS $\Sigma$ is average observable if

$$
\operatorname{rank}\left[\begin{array}{c}
\tilde{A}_{12} \\
Q^{\mathrm{T}}
\end{array}\right]=\operatorname{rank} \tilde{A}_{12}
$$

where $\tilde{A}_{12}$ is given in (2) and $Q=\operatorname{diag}\left[\mathbf{1}_{m_{2}}, \cdots, \mathbf{1}_{m_{r}}\right]$ is the characteristic matrix of the clustering $\mathcal{Q}$ of unmeasured nodes.

Proof: We show that (9) satisfies Proposition 1. First, to prove Proposition 1(i), assume (9) holds. Then, there exists $N=\left[\begin{array}{ll}N_{1} & N_{2}\end{array}\right] \in \mathbb{R}^{\ell \times \ell}$ with $N_{2}=0$ such that $N F=$ $N_{1} \tilde{A}_{12}=V Q^{\mathrm{T}}$, where $N_{1} \in \mathbb{R}^{\ell \times k}$ and $V \in \mathbb{R}^{\ell \times(r-1)}$. Therefore, for all $t \geq 0, N F \boldsymbol{\sigma}(t)=V Q^{\mathrm{T}} \boldsymbol{\sigma}(t)=0$.

Second, to prove Proposition 1(ii), we show that

$$
\begin{aligned}
\operatorname{rank}\left[\begin{array}{c}
s N-N E \\
H
\end{array}\right] & =\operatorname{rank}\left[\begin{array}{cc}
s N_{1}-N E_{1} & -V \Lambda^{-1} \\
I_{k} & 0
\end{array}\right] \\
& =k+\operatorname{rank}\left(V \Lambda^{-1}\right)=k+r-1,
\end{aligned}
$$

for all $s \in \mathbb{C}$, where $E=\left[\begin{array}{ll}E_{1} & F Q\end{array}\right], N=\left[\begin{array}{ll}N_{1} & 0\end{array}\right]$, and $N E=\left[\begin{array}{ll}N E_{1} & V \Lambda^{-1}\end{array}\right]$. Note that $\operatorname{rank}\left(V \Lambda^{-1}\right)=\operatorname{rank} V$ since $\Lambda^{-1}=Q^{\mathrm{T}} Q$ is a diagonal matrix with positive diagonal entries. Therefore, for Proposition 1(ii) to hold, it is necessary and sufficient that rank $V=r-1$, i.e., full column rank. We have, by definition,

$$
\left[\begin{array}{c}
\tilde{A}_{12} \\
Q^{\mathrm{T}}
\end{array}\right]=\left[\begin{array}{cccc}
A_{12} & A_{13} & \cdots & A_{1 r} \\
\mathbf{1}^{\mathrm{T}} & 0 & \cdots & 0 \\
0 & \mathbf{1}^{\mathrm{T}} & \cdots & 0 \\
\vdots & \vdots & \ddots & \vdots \\
0 & 0 & \cdots & \mathbf{1}^{\mathrm{T}}
\end{array}\right],
$$

where $\tilde{A}_{12} \in \mathbb{R}^{k \times m}$ and $A_{1 h} \in \mathbb{R}^{k \times m_{h}}$, for $h=2, \cdots, r$, are given in (2). Thus, it holds that

$$
\operatorname{rank}\left[\begin{array}{c}
\tilde{A}_{12} \\
Q^{\mathrm{T}}
\end{array}\right]=\sum_{h=2}^{r} \operatorname{rank}\left[\begin{array}{c}
A_{1 h} \\
\mathbf{1}^{\mathrm{T}}
\end{array}\right] .
$$

If (9) holds, then

$$
\operatorname{rank} \tilde{A}_{12}=\sum_{h=2}^{r} \operatorname{rank}\left[\begin{array}{c}
A_{1 h} \\
\mathbf{1}^{\mathrm{T}}
\end{array}\right]
$$

and there exists $\mathbf{p}_{1 h} \in \mathbb{R}^{k}$ such that $\mathbf{p}_{1 h}^{\mathrm{T}} A_{1 h}=\mathbf{1}_{m_{h}}^{\mathrm{T}}$ and $\mathbf{p}_{1 h}^{\mathrm{T}} A_{1 g}=0$, for all $h, g=2, \cdots, r$ and $h \neq g$. Therefore, we can choose $N_{1}=\left[\begin{array}{llllll}\mathbf{p}_{12} & \mathbf{p}_{13} & \cdots & \mathbf{p}_{1 r} & 0_{k \times \ell}\end{array}\right]^{\mathrm{T}}$ such that $N_{1} \tilde{A}_{12}=V Q^{\mathrm{T}}$, where $V=\left[\begin{array}{ll}I_{r-1} & 0_{(r-1) \times k}\end{array}\right]^{\mathrm{T}}$ with rank $V=r-1$. This concludes the proof.

Based on (9), we can provide a closed-form expression for reconstructing the average states of the clusters.

Corollary 3.1: Suppose a CNS $\Sigma$ that satisfies the sufficient condition (9) of AO. Then, the average states of the clusters are given by

$$
\mathbf{z}_{2}(t)=\Lambda Q^{\mathrm{T}} \tilde{A}_{12}^{+} \widehat{\mathbf{y}}(t),
$$

where $\mathbf{z}_{2}(t)=\left[x_{2}^{\mathrm{av}}(t) \cdots x_{h}^{\mathrm{av}}(t)\right]^{\top}, \Lambda=\left(Q^{\top} Q\right)^{-1}$, and $\widehat{\mathbf{y}}(t)=\dot{\mathbf{y}}(t)-A_{11} \mathbf{y}(t)-B_{1} \mathbf{u}(t)$.
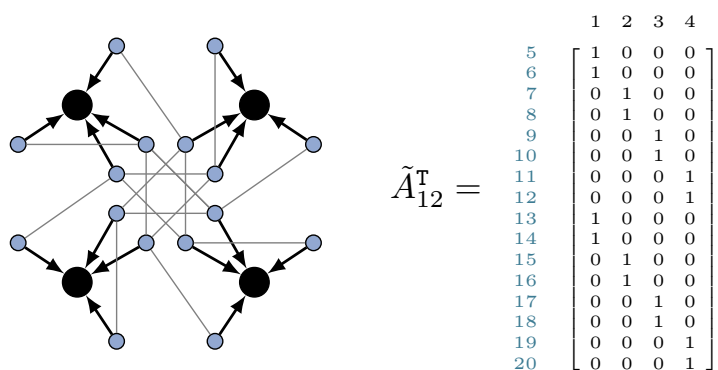

Fig. 2: Example of a CNS with a single cluster of unmeasured nodes (shown as blue) that is average observable, where the measured nodes (shown as black) span all the unmeasured nodes.

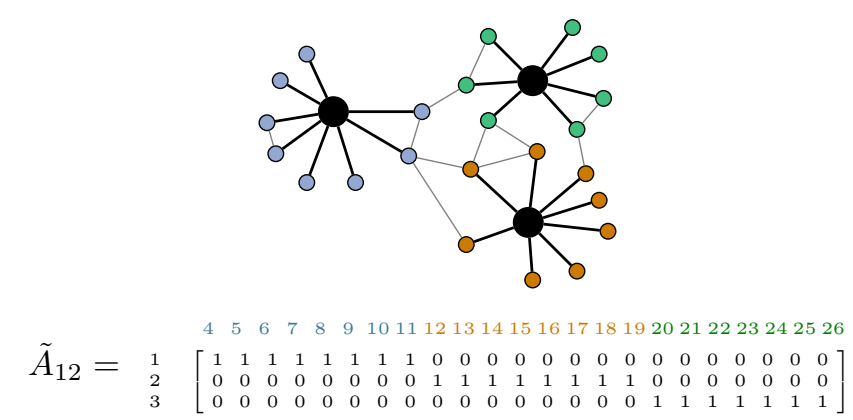

Fig. 3: Example of a CNS with three clusters of unmeasured nodes (shown as blue, green, and brown). The network is average observable with the dedicated state measurements at the hubs (shown as black) that respectively span distinct clusters of unmeasured nodes.

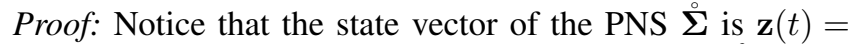
$\left[\begin{array}{ll}\mathbf{y}^{\top}(t) & \mathbf{z}_{2}^{\top}(t)\end{array}\right]^{\top}$, and the derivative of its output of $\mathbf{\Sigma}^{\circ}$ is

$\dot{\mathbf{y}}(t)=H \dot{\mathbf{z}}(t)=H\left[E_{1} \mathbf{y}(t)+F Q \mathbf{z}_{2}(t)+F \boldsymbol{\sigma}(t)+G \mathbf{u}(t)\right] ;$

where $H E_{1}=A_{11}, H F=\tilde{A}_{12}$, and $H G=B_{1}$. Recall that the output $\mathbf{y}(t)$ and the input $\mathbf{u}(t)$ trajectories are assumed to be known for all $t \geq 0$. Thus, theoretically, in the sense of Kalman's observability, the derivative $\dot{\mathbf{y}}(t)$ can be computed. Therefore, $\widehat{\mathbf{y}}(t):=\dot{\mathbf{y}}(t)-A_{11} \mathbf{y}(t)-B_{1} \mathbf{u}(t)$ is a known quantity, and $\widehat{\mathbf{y}}(t)=\tilde{A}_{12} Q \mathbf{z}_{2}(t)+\tilde{A}_{12} \boldsymbol{\sigma}(t)$. Now, if (9) holds, then there exists $N_{1} \in \mathbb{R}^{\ell \times k}$ such that $N_{1} \tilde{A}_{12}=V Q^{\top}$, where $V=\left[\begin{array}{ll}I_{r-1} & 0_{(r-1) \times k}\end{array}\right]^{\mathrm{T}}$, as shown in the proof of Theorem 3. Therefore, for all $t \geq 0, N_{1} \tilde{A}_{12} \boldsymbol{\sigma}(t)=0$, where $N_{1}=V Q^{\mathrm{T}} \tilde{A}_{12}^{+}$with $\tilde{A}_{12} \tilde{A}_{12}^{+}=\bar{I}_{k}$. Thus, we have

$$
N_{1} \widehat{\mathbf{y}}(t)=N_{1} \tilde{A}_{12} Q \mathbf{z}_{2}(t) \Rightarrow V Q^{\mathrm{T}} \tilde{A}_{12}^{+} \widehat{\mathbf{y}}(t)=V Q^{\mathrm{T}} Q \mathbf{z}_{2}(t) .
$$

Realizing that $V^{\mathrm{T}} V=I_{r-1}$ and that $Q^{\mathrm{T}} Q$ is invertible, we obtain (10).

\section{B. Graph-theoretic interpretation}

We provide a graph-theoretic interpretation to the sufficient condition (9) of average observability, which only concerns the induced bipartite subgraphs $\mathcal{G}_{1 h}$, for $h=2, \cdots, r$, which capture the topology of directed edges from unmeasured to 
measured nodes. This is quite intuitive because the information received by the measured nodes from the clusters of unmeasured nodes is crucial for average observability.

Consider the example of Fig. 2 with a single cluster $\mathcal{C}_{2}$ of unmeasured nodes, i.e., $r=2$ and $Q=\mathbf{1}_{m_{2}}$. For the condition (9) to hold, it is necessary that all the columns of $\tilde{A}_{12}$ are nonzero. In other words, for all $j \in \mathcal{C}_{2}$, there must exist an edge $(i, j) \in \mathcal{E}$, where $i \in \mathcal{C}_{1}$ is the measured node. That is to say, the measured nodes, at least minimally, cover (or span) all the unmeasured nodes. Since the graph in Fig. 2 satisfies this condition, therefore it is average observable. This can be verified by observing that $Q^{\mathrm{T}}=\mathbf{1}^{\mathrm{T}}$ is in the rowspace of $\tilde{A}_{12}$ (given in Fig. 2).

We can directly extend the single cluster interpretation to the case of multiple clusters of unmeasured nodes. That is, every measured node spans a distinct cluster of unmeasured nodes. This is illustrated by a CNS shown in Fig. 3, where the hubs are the measured nodes and the three clusters of unmeasured nodes are shown with different colors. For this network, we have $Q=\operatorname{diag}\left[\mathbf{1}_{8}, \mathbf{1}_{8}, \mathbf{1}_{7}\right]$ which equals $\tilde{A}_{12}^{\mathrm{T}}$ (given in Fig. 3 ). Therefore, (9) is satisfied.

\section{Average state observer}

Average observability is a property of $\boldsymbol{\Sigma}$ that allows for the reconstruction of the average states of given clusters of nodes in $\boldsymbol{\Sigma}$. It is, however, a theoretical notion. That is, in practice, the closed-form solution (10) should not be used because it requires the derivative of the output, which is very sensitive to the measurement noise.

To reconstruct the average states of clusters in real-time, we consider an average state observer of the form:

$$
\widehat{\boldsymbol{\Omega}}:\left\{\begin{array}{l}
\dot{\mathbf{w}}(t)=M \mathbf{w}(t)+K \mathbf{y}(t)+N G \mathbf{u}(t) \\
\boldsymbol{\zeta}(t)=\mathbf{w}(t)+L \mathbf{y}(t)
\end{array},\right.
$$

where $M \in \mathbb{R}^{(r-1) \times(r-1)}, N \in \mathbb{R}^{(r-1) \times \ell}$ and $K, L \in$ $\mathbb{R}^{(r-1) \times k}$, and $\mathbf{w}(t) \in \mathbb{R}^{r-1}$ is the state of $\widehat{\Omega}$ and $\boldsymbol{\zeta}(t) \in \mathbb{R}^{r-1}$ is the estimate of the average states. Let $\tilde{\mathbf{e}}_{\zeta}(t):=\mathbf{z}_{2}(t)-\boldsymbol{\zeta}(t)$ be the estimation error, where $\mathbf{z}_{2}(t)=\left[x_{2}^{\mathrm{av}}(t) \cdots x_{h}^{\mathrm{av}}(t)\right]^{\top}$ is the vector of average states of clusters. Then, we have

$$
\begin{aligned}
\dot{\tilde{\mathbf{e}}}_{\zeta}= & \left(\Lambda Q^{\mathrm{T}} \tilde{A}_{22} Q-L \tilde{A}_{12} Q\right) \tilde{\mathbf{e}}_{\zeta}+\left(\Lambda Q^{\mathrm{T}} \tilde{A}_{22} Q-L \tilde{A}_{12} Q-M\right) \mathbf{w} \\
& +\left[\left(\Lambda Q^{\mathrm{T}} \tilde{A}_{22} Q-L \tilde{A}_{12} Q\right) L-L A_{11}+\Lambda Q^{\mathrm{T}} \tilde{A}_{21}-K\right] \mathbf{y} \\
& +\left(\Lambda Q^{\mathrm{T}} \tilde{B}_{2}-L B_{1}-N G\right) \mathbf{u}+\left(\Lambda Q^{\mathrm{T}} \tilde{A}_{22}-L \tilde{A}_{12}\right) \boldsymbol{\sigma} .
\end{aligned}
$$

Thus, we can choose $M, K, N$ matrices as follows

$$
\begin{aligned}
M & =\Lambda Q^{\mathrm{T}} \tilde{A}_{22} Q-L \tilde{A}_{12} Q \\
K & =M L-L A_{11}+\Lambda Q^{\mathrm{T}} \tilde{A}_{21} \\
N & =\left[\begin{array}{cc}
-L & I_{r-1}
\end{array}\right],
\end{aligned}
$$

where $L$ is such that, for some $V_{2} \in \mathbb{R}^{(r-1) \times(r-1)}$,

$$
\begin{aligned}
& N F=\Lambda Q^{\mathrm{T}} \tilde{A}_{22}-L \tilde{A}_{12}=V_{2} Q^{\mathrm{T}}, \\
& M=\Lambda Q^{\mathrm{T}} \tilde{A}_{22} Q-L \tilde{A}_{12} Q \text { is Hurwitz. }
\end{aligned}
$$

The design criteria (12a) is to cancel the effect of $\sigma$ from the error dynamics, and (12b) is to ensure the exponential convergence of the error to zero. Choosing $M, K, N$ as in (11), we obtain the error dynamics as

$$
\dot{\tilde{\mathbf{e}}}_{\zeta}(t)=M \tilde{\mathbf{e}}_{\zeta}(t)+N F \boldsymbol{\sigma}(t) .
$$

Note that $M=N F Q$. We say that the average state observer $\widehat{\Omega}$ exists if (12) holds and the pair $\left(\tilde{A}_{12} Q, \Lambda Q^{\mathrm{T}} \tilde{A}_{22} Q\right)$ is observable. That is, we have $\tilde{\mathbf{e}}_{\zeta}(t) \rightarrow 0$ as $t \rightarrow \infty$ exponentially and at an arbitrary rate. Due to the ability to adjust the rate of convergence arbitrarily by the appropriate choice of $M$, the term 'tunable' average observer can be used for $\widehat{\Omega}$, [33].

Theorem 4: The average state observer $\widehat{\Omega}$ exists if and only if

$$
\operatorname{rank}\left[\begin{array}{c}
\tilde{A}_{12} \\
\Lambda Q^{\mathrm{T}} \tilde{A}_{22} \\
Q^{\mathrm{T}}
\end{array}\right]=\operatorname{rank} \tilde{A}_{12}
$$

where $Q \in \mathbb{R}^{m \times(r-1)}$ is the characteristic matrix of $\mathcal{Q}$ and $\Lambda=\left(Q^{\mathrm{T}} Q\right)^{-1}$.

Proof: (Sufficiency) Assume (14) holds. Then,

$$
L=\left(\Lambda Q^{T} \tilde{A}_{22}-V_{2} Q^{\mathrm{T}}\right) \tilde{A}_{12}^{+},
$$

where $\tilde{A}_{12}^{+}$is the pseudoinverse of $\tilde{A}_{12}$, is the solution to (12a) if and only if

$$
\operatorname{rank}\left[\begin{array}{c}
\tilde{A}_{12} \\
\Lambda Q^{\mathrm{T}} \tilde{A}_{22}-V_{2} Q^{\mathrm{T}}
\end{array}\right]=\operatorname{rank} \tilde{A}_{12},
$$

for some $V_{2} \in \mathbb{R}^{(r-1) \times(r-1)}$. From this, it is clear that if (14) holds, then (16) holds for all $V_{2} \in \mathbb{R}^{(r-1) \times(r-1)}$. Thus, $N F=V_{2} Q^{\mathrm{T}}$ and $M=N F Q=V_{2} Q^{\mathrm{T}} Q$, where $Q^{\mathrm{T}} Q=\Lambda^{-1}$. Then, let $V_{2}=W \Lambda$, where $W$ is an arbitrary Hurwitz matrix. Therefore, (12b) is satisfied since $M=W$, and $\tilde{\mathbf{e}}_{\zeta}(t) \rightarrow 0$ as $t \rightarrow \infty$ at an arbitrary rate since the eigenvalues of $W$ can be chosen arbitrarily.

(Necessity) Assume $\widehat{\Omega}$ exists, then (12) holds and $M$ can be assigned arbitrary eigenvalues. In other words, (16) holds for all $V_{2}=M \Lambda$, where $M \in \mathbb{R}^{(r-1) \times(r-1)}$ is any Hurwitz matrix with arbitrary eigenvalues. Let $M_{1} \in \mathbb{R}^{(r-1) \times(r-1)}$ and $M_{2} \in \mathbb{R}^{(r-1) \times(r-1)}$ be two Hurwitz matrices of full rank such that all the corresponding rows (or columns) of $M_{1}$ and $M_{2}$ are pairwise linearly independent. That is, row- $i$ of $M_{1}$ is linearly independent from row- $i$ of $M_{2}$, for $i=1, \cdots, r-1$. By assumption, (16) holds for both $V_{2}=M_{1} \Lambda$ and $V_{2}=M_{2} \Lambda$, which implies

$$
\operatorname{rank}\left[\begin{array}{c}
\tilde{A}_{12} \\
\Lambda Q^{\mathrm{T}} \tilde{A}_{22}-M_{1} \Lambda Q^{\mathrm{T}} \\
\Lambda Q^{\mathrm{T}} \tilde{A}_{22}-M_{2} \Lambda Q^{\mathrm{T}}
\end{array}\right]=\operatorname{rank} \tilde{A}_{12} .
$$

Using the fact that the corresponding rows (or columns) of $M_{1}$ and $M_{2}$ are linearly independent and $\operatorname{rank}\left(M_{1}\right)=\operatorname{rank}\left(M_{2}\right)=$ $r-1$, we have

$$
\begin{aligned}
\operatorname{rank}\left[\begin{array}{cc}
I & -M_{1} \Lambda \\
I & -M_{2} \Lambda
\end{array}\right] & =\operatorname{rank}\left[\begin{array}{cc}
I & -M_{1} \\
I & -M_{2}
\end{array}\right]\left[\begin{array}{cc}
I & 0 \\
0 & \Lambda
\end{array}\right] \\
& =\operatorname{rank}\left[\begin{array}{cc}
I & -M_{1} \\
I & -M_{2}
\end{array}\right] \\
& =2(r-1)
\end{aligned}
$$

That is, the above matrix is invertible, which implies that

$$
\text { the matrix }\left[\begin{array}{ccc}
I & 0 & 0 \\
0 & I & -M_{1} \Lambda \\
0 & I & -M_{2} \Lambda
\end{array}\right] \text { is invertible. }
$$


Finally, notice that

$$
\left[\begin{array}{c}
\tilde{A}_{12} \\
\Lambda Q^{\mathrm{T}} \tilde{A}_{22}-M_{1} \Lambda Q^{\mathrm{T}} \\
\Lambda Q^{\mathrm{T}} \tilde{A}_{22}-M_{2} \Lambda Q^{\mathrm{T}}
\end{array}\right]=\left[\begin{array}{ccc}
I & 0 & 0 \\
0 & I & -M_{1} \Lambda \\
0 & I & -M_{2} \Lambda
\end{array}\right]\left[\begin{array}{c}
\tilde{A}_{12} \\
\Lambda Q^{\mathrm{T}} \tilde{A}_{22} \\
Q^{\mathrm{T}}
\end{array}\right],
$$

therefore,

$$
\operatorname{rank}\left[\begin{array}{c}
\tilde{A}_{12} \\
\Lambda Q^{\mathrm{T}} \tilde{A}_{22}-M_{1} \Lambda Q^{\mathrm{T}} \\
\Lambda Q^{\mathrm{T}} \tilde{A}_{22}-M_{2} \Lambda Q^{\mathrm{T}}
\end{array}\right]=\operatorname{rank}\left[\begin{array}{c}
\tilde{A}_{12} \\
\Lambda Q^{\mathrm{T}} \tilde{A}_{22} \\
Q^{\mathrm{T}}
\end{array}\right] .
$$

From (17) and (18), we obtain (14).

Example 1: Consider a linear compartmental system, [25], with the dynamics at each node $j$ given by

$$
\dot{x}_{j}(t)=\sum_{i \in \mathcal{N}_{j}^{\downarrow}} a_{j i} x_{i}(t)-\sum_{o \in \mathcal{N}_{j}^{\uparrow}} a_{o j} x_{j}(t)+\sum_{l=1}^{2} b_{j l} u_{l}(t),
$$

where $a_{j i}=1$ and $a_{o j}=1$ for all $j \in \mathcal{V}, i \in \mathcal{N}_{j}^{\downarrow}$, and $o \in \mathcal{N}_{j}^{\uparrow}$. Note that $b_{j l}=1$ if $u_{l}(t)$ is applied on $j$, and $b_{j l}=0$ otherwise. Let the system be defined on the graph shown in Fig. 2, where the measured nodes are shown as black and the unmeasured nodes as blue. We consider a single cluster of unmeasured nodes, i.e., $r=2$ and $Q=\mathbf{1}_{16}$, and estimate its average state $\mathbf{z}_{2}(t)=x_{2}^{\text {av }}(t)$. Let the input vector be $\mathbf{u}(t)=\left[\begin{array}{l}u_{1}(t) \\ u_{2}(t)\end{array}\right]=\left[\begin{array}{c}\sin (t)+5 \\ \sin (3 t)\end{array}\right]$ and the input matrix $B=\left[\begin{array}{c}B_{1} \\ \tilde{B}_{2}\end{array}\right]$ with $B_{1}=0$ and $\tilde{B}_{2}=\left[\begin{array}{cc}\mathbf{1}_{8} & 0 \\ 0 & \mathbf{1}_{8}\end{array}\right]$. The output matrix $C=\left[\begin{array}{ll}I_{4} & 0\end{array}\right]$. Note that $\Lambda Q^{\mathrm{T}} \widetilde{A}_{22}=-\frac{1}{16} \mathbf{1}_{16}^{\mathrm{T}}$ since the internal outflow centrality of each blue node with respect to the induced subgraph $\mathcal{G}_{22}$ is -1 and $\Lambda=-1 / 16$. Thus, in this example, the condition (9) and (14) are equivalent, i.e., the system is $\mathrm{AO}$ and $\widehat{\boldsymbol{\Omega}}$ exists. The parameters of $\widehat{\boldsymbol{\Omega}}$, for $V_{2}=-3 / 64$, are computed from (11) and (15) as

$$
\begin{aligned}
& M=-0.75, \quad N=\left[\begin{array}{ll}
\frac{1}{64} \mathbf{1}_{4}^{\mathrm{T}} & 1
\end{array}\right], \\
& K=\frac{3}{256} \mathbf{1}_{4}^{\mathrm{T}}, \quad L=-\frac{1}{64} \mathbf{1}_{4}^{\mathrm{T}} .
\end{aligned}
$$

The average state can be estimated asymptotically by the observer $\widehat{\Omega}$, as shown in Fig. 4, where the rate of convergence can be set by choosing different values of $V_{2}$.

Remark 3: Similar to the functional observer of Darouach [4], the average state observer $\widehat{\Omega}$ has a minimum dimension, i.e., it equals the number of unmeasured clusters $r-1$. Following the literature [21]-[23] of functional observers, however, one can design an average state observer of higher dimension if (14) is not satisfied. The dimension of the observer is determined iteratively. For instance, to reconstruct $\mathbf{z}_{2}(t)=\hat{P} \mathbf{x}(t)$, where $\hat{P}=\left[\begin{array}{ll}0_{(r-1) \times k} & \Lambda Q^{\mathrm{T}}\end{array}\right]$, we can employ the design procedure of [23], where the observer is of dimension $v(r-1)$ with $v=1,2,3, \cdots$ such that

$$
\operatorname{rank}\left[\begin{array}{c}
\hat{P} A^{v} \\
\mathcal{O}_{C, A, v} \\
\mathcal{O}_{\hat{P}, A, v}
\end{array}\right]=\operatorname{rank}\left[\begin{array}{c}
\mathcal{O}_{C, A, v} \\
\mathcal{O}_{\hat{P}, A, v}
\end{array}\right]
$$

with $\mathcal{O}_{C, A, v}=\left[\begin{array}{llll}C^{\mathrm{T}} & (C A)^{\mathrm{T}} & \cdots & \left(C A^{v-1}\right)^{\mathrm{T}}\end{array}\right]^{\mathrm{T}}$ and $\mathcal{O}_{\hat{P}, A, v}=\left[\begin{array}{llll}\hat{P}^{\mathrm{T}} & (\hat{P} A)^{\mathrm{T}} & \cdots & \left(\hat{P} A^{v-1}\right)^{\mathrm{T}}\end{array}\right]^{\mathrm{T}}$. However, to

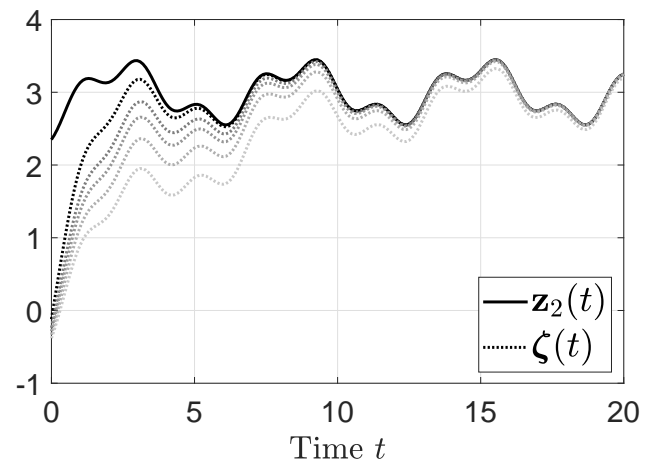

Fig. 4: Asymptotic estimation of the average state of a cluster of unmeasured nodes in the graph shown in Fig. 2 by $\widehat{\Omega}$. The solid line shows the average state trajectory of the cluster of unmeasured nodes, and the dotted line shows the estimation of the average state at different rates. Different rates of convergence are set by choosing $V_{2}=-3 / 64,-1 / 32,-1 / 40,-3 / 160,-1 / 80$ from faster to slower, respectively.

determine $v$, we have to compute (20) iteratively, which is not computationally feasible for large-scale systems. Moreover, the dimension scales with $v$, which may yield an observer of very high dimension. Therefore, it is computationally feasible to design $\widehat{\Omega}$ of order $r-1$ by choosing $V_{2} \in \mathbb{R}^{(r-1) \times(r-1)}$ in (11) and (15) such that $\lim \sup _{t \rightarrow \infty}\left\|\tilde{\mathbf{e}}_{\zeta}(t)\right\|$ is minimum, [34], under the assumption that $\|\boldsymbol{\sigma}(t)\|$ is bounded for all $t \geq 0$. $\diamond$

\section{Average Detectability}

If the clustered network system $\Sigma$ is not average observable, the average states of given clusters $\mathcal{C}_{2}, \cdots, \mathcal{C}_{r}$ can still be asymptotically estimated if all the average states are stable. Such a property can be characterized as average detectability of a CNS.

Assume $\mathbf{u}(t)=0$ for all $t \geq 0$. Then, the CNS $\boldsymbol{\Sigma}$ with a given set of measured nodes $\mathcal{C}_{1}$ and a clustering $\mathcal{Q}=\left\{\mathcal{C}_{2}, \cdots, \mathcal{C}_{r}\right\}$ of unmeasured nodes is said to be average detectable (AD) if, for all $t \geq 0$ and $\mathbf{z}(0)=\mathbf{z}_{0} \in \mathbb{R}^{\ell}, \mathbf{y}(t)=0$ implies that the average states $x_{h}^{\text {av }}(t)$ in $\boldsymbol{\Sigma}_{\mathbf{\Sigma}}$ converge to zero as $t \rightarrow \infty$, where $h=2, \cdots, r$. That is, $\mathbf{z}_{2}(t) \rightarrow 0$ as $t \rightarrow \infty$ when $\mathbf{y}(t)=0$ for all $t \geq 0$ and $\mathbf{z}_{2}(0) \in \mathbb{R}^{r-1}$, where $\mathbf{z}_{2}(t)=\left[x_{2}^{\mathrm{av}}(t) \cdots x_{r}^{\mathrm{av}}\right]^{\mathrm{T}}$.

\section{A. Necessary and sufficient condition}

Theorem 5: Assume that $\lim _{t \rightarrow \infty}\|\boldsymbol{\sigma}(t)\| \neq 0$. Then, the CNS $\boldsymbol{\Sigma}$ is average detectable if and only if the following two conditions hold:

(i) $\Lambda Q^{\mathrm{T}} \tilde{A}_{22} Q$ is Hurwitz.

(ii) $Q^{\mathrm{T}} \tilde{A}_{22}=\tilde{V} Q^{\mathrm{T}}$ for some $\tilde{V} \in \mathbb{R}^{(r-1) \times(r-1)}$.

Here, $\tilde{A}_{22}$ is given in (2), $Q \in \mathbb{R}^{m \times(r-1)}$ is the characteristic matrix of the clustering $\mathcal{Q}$, and $\Lambda=\left(Q^{\mathrm{T}} Q\right)^{-1}$.

Proof: Let $R=\Lambda Q^{\mathrm{T}} \tilde{A}_{22}$.

(Sufficiency) The dynamics of $\mathbf{z}_{2}(t)$ from $\stackrel{\circ}{\Sigma}^{\circ}$ is given as

$$
\dot{\mathbf{z}}_{2}(t)=R Q \mathbf{z}_{2}(t)+R \boldsymbol{\sigma}(t)+\Lambda Q^{\mathrm{T}}\left[\tilde{A}_{21} \mathbf{y}(t)+\tilde{B}_{2} \mathbf{u}(t)\right] .
$$



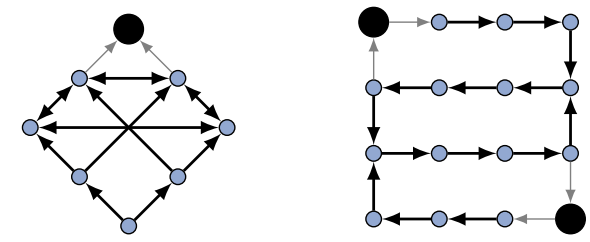

Fig. 5: Examples of CNSs that are average detectable with a single cluster of unmeasured nodes. The measured nodes $\mathcal{C}_{1}$ are shown as black. The local feedback gains $a_{i i}=-3$, for all $i \in \mathcal{C}_{2}$, where $\mathcal{C}_{2}$ are the unmeasured nodes (blue). The subgraph $\mathcal{G}_{22}$, highlighted by darker edges, is negatively balanced in both networks.

The solution, when $\mathbf{u}(t)=0$ and $\mathbf{y}(t)=0$ for all $t \geq 0$, is

$$
\mathbf{z}_{2}(t)=\exp (R Q t) \mathbf{z}_{2}(0)+\int_{0}^{t} \exp [R Q(t-\tau)] R \boldsymbol{\sigma}(\tau) d \tau \text {. }
$$

If (i) holds, then $\exp (R Q t) \rightarrow 0$ as $t \rightarrow \infty$. If (ii) holds, then $R \boldsymbol{\sigma}(t)=0$ for all $t \geq 0$ since $Q^{\mathrm{T}} \boldsymbol{\sigma} \equiv 0$. Hence, $\mathbf{z}_{2}(t) \rightarrow 0$ as $t \rightarrow \infty$ for all $\mathbf{z}_{2}(0) \in \mathbb{R}^{r-1}$.

(Necessity) Assume (i) doesn't hold, then $R Q$ is not Hurwitz, $\exp (R Q t) \rightarrow \infty$ as $t \rightarrow \infty$, and, therefore, $\mathbf{z}_{2}(t) \rightarrow \infty$. Now, assume (i) holds but (ii) doesn't hold, then we have

$$
\lim _{t \rightarrow \infty}\left\|\mathbf{z}_{2}(t)\right\|=\lim _{t \rightarrow \infty}\left\|\int_{0}^{t} \exp (R Q \eta) R \boldsymbol{\sigma}(t-\eta) d \eta\right\|,
$$

which must be 0 for average detectability. Since we assume that $\lim _{t \rightarrow \infty}\|\boldsymbol{\sigma}(t)\| \neq 0$ and (ii) doesn't hold, we have $\lim _{t \rightarrow \infty}\left\|\mathbf{z}_{2}(t)\right\|=0$ only if $\exp (R Q t) R=0$ for all $t \geq 0$. This is not possible because it requires $R \in \operatorname{ker}(\exp (R Q t))$, where $\operatorname{ker}(\exp (R Q t))=\emptyset$ for all $t \geq 0$, i.e., $\exp (R Q t)$ is always non-singular (see Ch. 5, Sec. 5.6, Prob. 43 in [35]). This proves the necessity of (ii).

\section{B. Graph-theoretic interpretation}

For a CNS with a single cluster of unmeasured nodes, AD requires certain regularity of the induced subgraph formed by unmeasured nodes. In such a case, the conditions in Theorem 5 boil down to $\mathbf{1}_{m_{2}}^{\mathrm{T}} \tilde{A}_{22}=-\gamma \mathbf{1}_{m_{2}}^{\mathrm{T}}$, where $\mathbf{1}_{m_{2}}=Q$ and $\gamma>0$. That is, for average detectability of a network system with a single cluster of unmeasured nodes, the induced subgraph $\mathcal{G}_{22}=\left(\mathcal{C}_{2}, \mathcal{E}_{22}\right)$ must be negatively balanced, i.e., the internal outflow centrality $\phi_{i, 2}$ of every node $i \in \mathcal{C}_{2}$ in (4) with respect to $\mathcal{G}_{22}$ must be equal and negative. This is illustrated by the graphs shown in Fig. 5, where the internal outflow centrality of every unmeasured node with respect to $\mathcal{G}_{22}$ in the left and right graph is -1 and -2 , respectively.

For a CNS with multiple clusters of unmeasured nodes, in addition to having each subgraph $\mathcal{G}_{h h}$ negatively balanced, we also require that the clustering $\mathcal{Q}$ is almost equitable (see [15], [16] for definition), which means that the relative outdegree $d_{i, g}$ in (5), for all $i \in \mathcal{C}_{h}$, with respect to cluster $\mathcal{C}_{g}$ is equal, for all $g \neq h$. This is illustrated with an unweighted graph shown in Fig. 6, where each of the subgraphs $\mathcal{G}_{h h}$ is negatively balanced for $h=2,3,4,5$ shown with red, green, brown, and blue nodes, respectively. It is because for all nodes

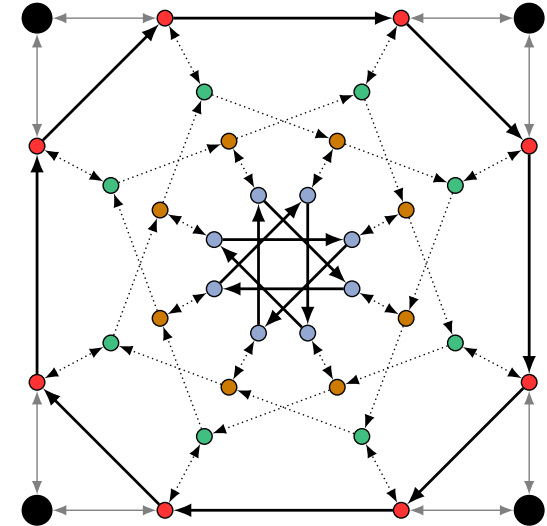

Fig. 6: Example of a CNS that is average detectable with measured nodes $\mathcal{C}_{1}$ shown as black and the four clusters of unmeasured nodes $\mathcal{C}_{2}, \mathcal{C}_{3}, \mathcal{C}_{4}, \mathcal{C}_{5}$ shown as red, green, brown, and blue, respectively. The local feedback gains $a_{i i}=\sum_{j \in \mathcal{N}_{i}^{\uparrow}} a_{j i}$. Each induced subgraph $\mathcal{G}_{h h}$, highlighted by darker edges, is negatively balanced. The clustering $\mathcal{Q}=\left\{\mathcal{C}_{2}, \mathcal{C}_{3}, \mathcal{C}_{4}, \mathcal{C}_{5}\right\}$ is almost equitable. The induced bipartite subgraphs $\mathcal{G}_{h g}$ are highlighted by dotted edges, for $h, g=2,3,4,5$ and $h \neq g$.

in red, green, brown and blue clusters, we have internal outflow centrality equal to $-2,-1,-2$ and -1 , respectively. Moreover, considering the induced bipartite subgraphs $\mathcal{G}_{h g}$, we see that the relative out-degree of all the nodes in a certain cluster are also equal. For instance, consider the induced bipartite subgraph $\mathcal{G}_{45}$ with directed edges from blue to brown nodes. Each blue node has relative out-degree with respect to brown nodes equal to 1 . Similarly, in both $\mathcal{G}_{35}$ and $\mathcal{G}_{25}$, the relative out-degree of blue nodes with respect to $\mathcal{C}_{3}$ and $\mathcal{C}_{2}$ is 0 . Therefore, the clustering of unmeasured nodes $\mathcal{Q}$ is almost equitable, which is also known as externally equitable [14] when the graph is unweighted. Hence, the CNS shown in Fig. 6 is average detectable.

\section{Open-loop average state estimation}

Consider the following open-loop average state observer:

$$
\widehat{\boldsymbol{\Psi}}: \quad \dot{\boldsymbol{\theta}}(t)=R Q \boldsymbol{\theta}(t)+\Lambda Q^{\mathrm{T}}\left[\tilde{A}_{21} \mathbf{y}(t)+\tilde{B}_{2} \mathbf{u}(t)\right],
$$

where $R=\Lambda Q^{\mathrm{T}} \tilde{A}_{22}$. Let the estimation error be

$$
\tilde{\mathbf{e}}_{\theta}(t):=\mathbf{z}_{2}(t)-\boldsymbol{\theta}(t) .
$$

We say that the open-loop average state observer $\widehat{\Psi}$ exists if $\tilde{\mathbf{e}}_{\theta}(t) \rightarrow 0$ as $t \rightarrow \infty$.

Theorem 6: The open-loop average state observer $\widehat{\Psi}$ exists if and only if the CNS $\boldsymbol{\Sigma}$ is average detectable.

Proof: Consider $\tilde{\mathbf{e}}_{\theta}(t)=\mathbf{z}_{2}(t)-\boldsymbol{\theta}(t)$, then

$$
\dot{\tilde{\mathbf{e}}}_{\theta}(t)=R Q \tilde{\mathbf{e}}_{\theta}(t)+R \boldsymbol{\sigma}(t),
$$

whose solution is given as

$$
\tilde{\mathbf{e}}_{\theta}(t)=\exp (R Q t) \tilde{\mathbf{e}}_{\theta}(0)+\int_{0}^{t} \exp [R Q(t-\tau)] R \boldsymbol{\sigma}(\tau) d \tau \text {. }
$$



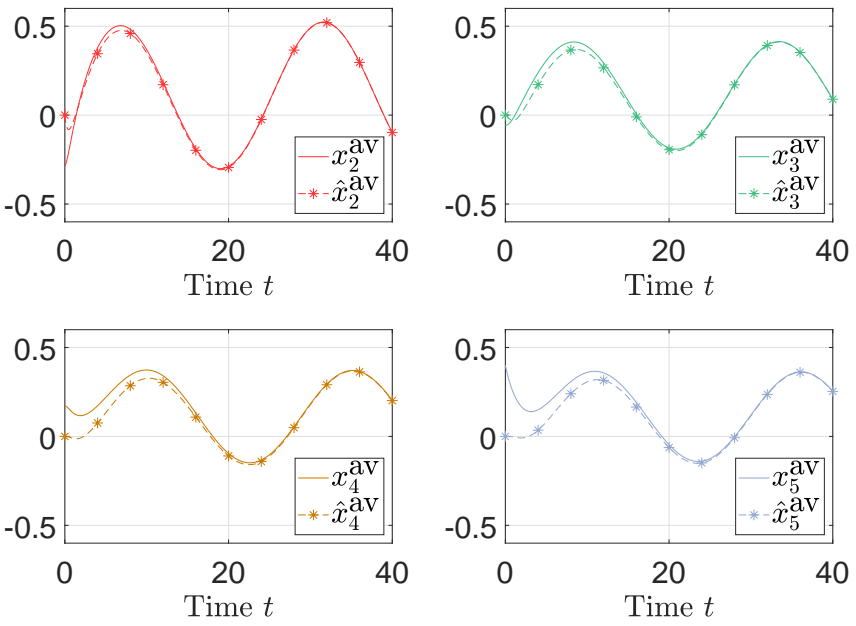

Fig. 7: Average state estimation of clusters of unmeasured nodes $\mathcal{C}_{2}, \mathcal{C}_{3}, \mathcal{C}_{4}, \mathcal{C}_{5}$ in a network shown in Fig. 6.

From here, the arguments for sufficiency and necessity follow the same lines of the proof of Theorem 5.

Note that Theorem 6 coincides with the strong detectability definition of [36], which considers arbitrary unknown inputs. However, as discussed earlier, $\boldsymbol{\sigma}(t)$ is not arbitrary.

Example 2: Consider an unweighted graph shown in Fig. 6, where measured nodes $\mathcal{C}_{1}=\{1,2,3,4\}$ are shown as black. The state of each node $j$ evolves according to (19). The input is given by

$$
u_{i}(t)= \begin{cases}\sin (0.5 t+(i-1) \pi / 4), & \text { if } i=1, \cdots, 4 \\ 0, & \text { otherwise }\end{cases}
$$

and the input matrix $B=\left[\begin{array}{ll}I_{4} & 0\end{array}\right]^{\mathrm{T}}$. Note that Theorem 5 (i) is satisfied since

$$
R Q=\left[\begin{array}{rrrr}
-2 & 1 & 0 & 0 \\
1 & -2 & 1 & 0 \\
0 & 1 & -2 & 1 \\
0 & 0 & 1 & -1
\end{array}\right], \quad \text { which is Hurwitz }
$$

where $R=\Lambda Q^{\mathrm{T}} \tilde{A}_{22}$. Also, Theorem 5 (ii) is satisfied since $Q^{\mathrm{T}} \tilde{A}_{22}=\Lambda^{-1} Q^{\mathrm{T}} \tilde{A}_{22} Q Q^{\mathrm{T}}$, where $Q=\operatorname{diag}\left[\mathbf{1}_{8}, \mathbf{1}_{8}, \mathbf{1}_{8}, \mathbf{1}_{8}\right]$. Therefore, the open-loop observer $\widehat{\Psi}$ converges to the average state of unmeasured clusters $\mathcal{C}_{2}, \mathcal{C}_{3}, \mathcal{C}_{4}, \mathcal{C}_{5}$ as shown in Fig. 7, where the initial states $\mathbf{x}(0)$ are chosen uniformly in the interval $(-0.5,0.5)$. The rate of convergence of the estimation error is determined by $\operatorname{eig}(R Q)=$ $\{-3.5321,-2.3473,-1.0000,-0.1206\}$, respectively.

Consensus case: The question we ask is that if the nodes in each of the clusters in $\mathcal{Q}=\left\{\mathcal{C}_{2}, \cdots, \mathcal{C}_{r}\right\}$ reach a consensus, [37], then "is $\Sigma$ average detectable?" Equivalently, if $\lim _{t \rightarrow \infty}\|\boldsymbol{\sigma}(t)\|=0$, then "does $\widehat{\boldsymbol{\Psi}}$ exist?"

Theorem 7: Assume that $R Q$ is a Hurwitz matrix. Then, $\widehat{\Psi}$ exists if $\lim _{t \rightarrow \infty}\|\boldsymbol{\sigma}(t)\|=0$.

Proof: Consider the estimation error $\tilde{\mathbf{e}}_{\theta}(t)=\mathbf{z}_{2}(t)-\boldsymbol{\theta}(t)$ with dynamics given in (22) and the trajectory given in (23). Since $R Q$ is assumed to be Hurwitz, therefore $\lim _{t \rightarrow \infty}\left\|\exp (R Q t) \tilde{\mathbf{e}}_{\theta}(0)\right\|=0$ for all $\tilde{\mathbf{e}}_{\theta}(0) \in \mathbb{R}^{r-1}$. Let

$$
\begin{aligned}
\mathbf{v}(t)= & \int_{0}^{t} \exp [R Q(t-\tau)] R \boldsymbol{\sigma}(\tau) d \tau \\
=\int_{0}^{t / 2} \exp [ & R Q(t-\tau)] R \boldsymbol{\sigma}(\tau) d \tau \\
& \quad+\int_{t / 2}^{t} \exp [R Q(t-\tau)] R \boldsymbol{\sigma}(\tau) d \tau \\
=\int_{t / 2}^{t} \exp ( & R Q \tau) R \boldsymbol{\sigma}(t-\tau) d \tau \\
& \quad+\int_{t / 2}^{t} \exp [R Q(t-\tau)] R \boldsymbol{\sigma}(\tau) d \tau
\end{aligned}
$$

and let $\|\cdot\| \|$ denote the matrix norm induced by $\|\cdot\|$, then, by the triangle and Cauchy-Schwarz inequalities, we have

$$
\begin{aligned}
\|\mathbf{v}(t)\| & \leq\left[\int_{t / 2}^{t}\|\exp (R Q \tau)\|^{2} d \tau\right]^{\frac{1}{2}}\left[\int_{t / 2}^{t}\|R \boldsymbol{\sigma}(t-\tau)\|^{2} d \tau\right]^{\frac{1}{2}} \\
& +\left[\int_{t / 2}^{t}\|\exp [R Q(t-\tau)]\|^{2} d \tau\right]^{\frac{1}{2}}\left[\int_{t / 2}^{t}\|R \boldsymbol{\sigma}(\tau)\|^{2} d \tau\right]^{\frac{1}{2}} .
\end{aligned}
$$

First, we have $\lim _{t \rightarrow \infty} \int_{t / 2}^{t}\|\| \exp (R Q \tau) \|^{2} d \tau=0$ since $R Q$ is Hurwitz. Second, we have $\lim _{t \rightarrow \infty} \int_{t / 2}^{t}\|R \boldsymbol{\sigma}(\tau)\|^{2} d \tau=0$ since $\lim _{t \rightarrow \infty}\|\boldsymbol{\sigma}(t)\|=0$. Thus, $\lim _{t \rightarrow \infty}\|\mathbf{v}(t)\|=0$, which implies $\lim _{t \rightarrow \infty}\left\|\tilde{\mathbf{e}}_{\theta}(t)\right\|=0$.

\section{CONCLUding REMARKS}

We addressed the problem of reconstruction of the average states of given clusters in a large-scale clustered network system (CNS). For computational tractability, we aggregated the clusters in CNS and obtained the projected network system (PNS). We defined average observability, which enables the reconstruction of the average states through the PNS, and provided its necessary and sufficient condition in Proposition 1. Then, from Proposition 1, we derived a necessary condition (Theorem 2) and a sufficient condition (Theorem 3) of average observability based on the structure of the CNS. We provided a graph-theoretic interpretation of Theorem 3 that concerns the topology between unmeasured nodes and measured nodes in the CNS. Average observability is essential for the design of a tunable average state observer that can reconstruct the average states of the clusters at an arbitrary rate (Theorem 4).

If the network system is not average observable, we can still asymptotically reconstruct the average states by an open-loop average state observer if the CNS is average detectable (Theorem 5 and 6). However, the rate at which the average states are estimated cannot be adjusted arbitrarily, and it depends on the eigenvalues of the state matrix of PNS. Moreover, in Theorem 7, we showed that if the consensus is reached in every cluster, the average states can be asymptotically estimated by the open-loop observer. On the other hand, if the consensus is not reached, then, in Theorem 5, the necessary and sufficient condition of average detectability requires a certain regularity not only in the induced subgraphs of the clusters, but also in the induced bipartite subgraphs that capture the inter-cluster topology.

The conditions derived in this paper provide a framework to study the problem of sensor location and cluster selection in large-scale network systems in order to achieve average observability and/or average detectability. 


\section{ACKNOWLEDGMENTS}

This work is supported by European Research Council (ERC) under the European Union's Horizon 2020 research and innovation programme (ERC-AdG no. 694209, ScaleFreeBack, website: http://scale-freeback.eu/). We are indebted to the anonymous reviewers whose comments led to a significant improvement of this paper.

\section{REFERENCES}

[1] A. C. Antoulas, Approximation of large-scale dynamical systems. Philadelphia, PA, USA: SIAM, 2005.

[2] D. Luenberger, "An introduction to observers," IEEE Transactions on Automatic Control, vol. 16, no. 6, pp. 596-602, 1971.

[3] P. Murdoch, "Observer design for a linear functional of the state vector," IEEE Transactions on Automatic Control, vol. 18, no. 3, pp. 308-310, 1973.

[4] M. Darouach, "Existence and design of functional observers for linear systems," IEEE Transactions on Automatic Control, vol. 45, no. 5, pp. 940-943, 2000.

[5] S. Coogan and M. Arcak, "A compartmental model for traffic networks and its dynamical behavior," IEEE Transactions on Automatic Control, vol. 60, no. 10, pp. 2698-2703, 2015.

[6] K. Deng, P. Barooah, P. G. Mehta, and S. P. Meyn, "Building thermal model reduction via aggregation of states," in Proceedings of the 2010 American Control Conference, 2010, pp. 5118-5123.

[7] W. Mei, S. Mohagheghi, S. Zampieri, and F. Bullo, "On the dynamics of deterministic epidemic propagation over networks," Annual Reviews in Control, vol. 44, pp. 116-128, 2017.

[8] G. A. Pagani and M. Aiello, "The power grid as a complex network: a survey," Physica A: Statistical Mechanics and its Applications, vol. 392, no. 11 , pp. $2688-2700,2013$.

[9] M. Aoki, "Control of large-scale dynamic systems by aggregation," IEEE Transactions on Automatic Control, vol. 13, no. 3, pp. 246-253, 1968.

[10] P. G. Coxson, "Lumpability and observability of linear systems," Journal of Mathematical Analysis and Applications, vol. 99, no. 2, pp. 435-446, 1984.

[11] F. M. Atay and L. Roncoroni, "Lumpability of linear evolution equations in banach spaces," Evolution Equations and Control Theory, vol. 6, no. 1, pp. 15-34, 2017.

[12] M. Ji and M. Egerstedt, "Observability and estimation in distributed sensor networks," in 2007 46th IEEE Conference on Decision and Control, 2007, pp. 4221-4226.

[13] S. Martini, M. Egerstedt, and A. Bicchi, "Controllability analysis of multi-agent systems using relaxed equitable partitions," International Journal of Systems, Control and Communications, vol. 2, no. 1-3, pp. 100-121, 2010.

[14] M. Egerstedt, S. Martini, M. Cao, K. Camlibel, and A. Bicchi, "Interacting with networks: How does structure relate to controllability in single-leader, consensus networks?" IEEE Control Systems Magazine, vol. 32, no. 4, pp. 66-73, 2012.

[15] N. Monshizadeh, H. L. Trentelman, and M. K. Camlibel, "Projectionbased model reduction of multi-agent systems using graph partitions," IEEE Transactions on Control of Network Systems, vol. 1, no. 2, pp. $145-154,2014$

[16] C. O. Aguilar and B. Gharesifard, "Almost equitable partitions and new necessary conditions for network controllability," Automatica, vol. 80, pp. 25-31, 2017.

[17] M. U. B. Niazi, X. Cheng, C. Canudas-de-Wit, and J. M. A. Scherpen, "Structure-based clustering algorithm for model reduction of large-scale network systems," in 2019 58th IEEE Conference on Decision and Control, 2019. [Online]. Available: https://hal.archives-ouvertes.fr/hal02278884

[18] M. Saeedmanesh and N. Geroliminis, "Clustering of heterogeneous networks with directional flows based on "Snake" similarities," Transportation Research Part B: Methodological, vol. 91, pp. 250-269, 2016.

[19] T. Ishizaki, K. Kashima, J.-i. Imura, and K. Aihara, "Model reduction and clusterization of large-scale bidirectional networks." IEEE Transactions on Automatic Control, vol. 59, no. 1, pp. 48-63, 2014.

[20] T. Sadamoto, T. Ishizaki, and J.-i. Imura, "Average state observers for large-scale network systems," IEEE Transactions on Control of Network Systems, vol. 4, no. 4, pp. 761-769, 2016.
[21] T. L. Fernando, H. M. Trinh, and L. Jennings, "Functional observability and the design of minimum order linear functional observers," IEEE Transactions on Automatic Control, vol. 55, no. 5, pp. 1268-1273, 2010.

[22] F. Rotella and I. Zambettakis, "A note on functional observability," IEEE Transactions on Automatic Control, vol. 61, no. 10, pp. 3197-3202, 2016.

[23] — "A direct design procedure for linear state functional observers," Automatica, vol. 70, pp. 211-216, 2016.

[24] M. U. B. Niazi, C. Canudas-de-Wit, and A. Y. Kibangou, "Average observability of large-scale network systems," in 2019 18th European Control Conference (ECC), 2019, pp. 1506-1511.

[25] G. G. Walter and M. Contreras, Compartmental modeling with networks. Springer-Birkhäuser Boston, 1999.

[26] C. Godsil and G. Royle, Algebraic Graph Theory. New York: SpringerVerlag, 2001.

[27] S. L. Campbell and C. D. Meyer, Generalized inverses of linear transformations. SIAM, 2009, vol. 56.

[28] F. J. Bejarano, L. Fridman, and A. Poznyak, "Unknown input and state estimation for unobservable systems," SIAM Journal Control Optimization, vol. 48 , no. 2 , pp. $1155-1178,2009$.

[29] H. L. Trentelman, A. A. Stoorvogel, and M. Hautus, Control theory for linear systems. Springer-Verlag London, 2002.

[30] E. Yip and R. Sincovec, "Solvability, controllability, and observability of continuous descriptor systems," IEEE Transactions on Automatic Control, vol. 26, no. 3, pp. 702-707, 1981.

[31] D. Cobb, "Controllability, observability, and duality in singular systems," IEEE Transactions on Automatic Control, vol. 29, no. 12, pp. 10761082, 1984.

[32] F. J. Bejarano, T. Floquet, W. Perruquetti, and G. Zheng, "Observability and detectability analysis of singular linear systems with unknown inputs," in 2011 50th IEEE Conference on Decision and Control and European Control Conference, 2011, pp. 4005-4010.

[33] G. Besançon, Nonlinear observers and applications. New York: Springer-Verlag, 2007.

[34] M. U. B. Niazi, D. Deplano, C. Canudas-de-Wit, and A. Y. Kibangou, "Scale-free estimation of the average state in large-scale systems," IEEE Control Systems Letters, vol. 4, no. 1, pp. 211-216, 2020.

[35] R. A. Horn and C. R. Johnson, Matrix analysis, 2nd ed. Cambridge university press, 2012.

[36] M. L. J. Hautus, "Strong detectability and observers," Linear Algebra and its Applications, vol. 50, pp. 353-368, 1983.

[37] R. Olfati-Saber, J. A. Fax, and R. M. Murray, "Consensus and cooperation in networked multi-agent systems," Proceedings of the IEEE, vol. 95, no. 1, pp. 215-233, 2007. 